\title{
The effect of physiotherapy and acupuncture on psychocognitive, somatic, quality of life, and disability characteristics in TTH patients
}

This article was published in the following Dove Press journal: Journal of Pain Research

\section{George Georgoudis ${ }^{1,2}$ \\ Bledjana Felah ${ }^{2}$ \\ Pantelis T Nikolaidis ${ }^{3}$ \\ Maria Papandreou' \\ Evanthia Mitsiokappa ${ }^{4}$ \\ Andreas F Mavrogenis ${ }^{4}$ \\ Thomas Rosemann ${ }^{5}$ \\ Beat Knechtle (5,6 $^{5}$}

'Musculoskeletal and Respiratory Research Lab, Department of Physiotherapy, University of West Attica, Athens, Greece; ${ }^{2}$ PhysioPain Group, Pain Specialist \& Rehabilitation Centers, Athens, Greece; ${ }^{3}$ Exercise Physiology Laboratory, Nikaia, Greece; ${ }^{4}$ First Department of Orthopaedics, National and Kapodistrian University of Athens, School of Medicine, Athens, Greece; ${ }^{5}$ Institute of Primary Care, University of Zurich, Zurich, Switzerland; ${ }^{\circ}$ Medbase St. Gallen Am Vadianplatz, St. Gallen, Switzerland
Correspondence: Beat Knechtle Medbase St. Gallen Am Vadianplatz, Vadianstrasse 26, 900 I St. Gallen, Switzerland

Tel +4I 07I 2269300

Fax +4| 07| 226 930 I

Email beat.knechtle@hispeed.ch
Introduction: Nonpharmacological therapies have been widely used to treat tension-type headache (TTH); however, limited evidence exists with regards to their effectiveness. Therefore, the aim of the present study was to examine the combined effect of acupuncture, stretching, and physiotherapy (myofascial release techniques and microwave diathermy) on psychocognitive, somatic, quality of life, and disability characteristics in such patients.

Subjects and methods: Patients with TTH $(n=44)$ performed either acupuncture or stretching (control group, $\mathrm{n}=20$ ) or acupuncture, stretching, and physiotherapy (experimental group, $\mathrm{n}=24$ ) during a 4 -week intervention period including 10 treatment sessions. They were tested for the Greek version of the Short-Form McGill Pain Questionnaire, the Greek version of the Migraine Disability Assessment Questionnaire, the Greek version of the Hospital Anxiety and Depression Scale, Short Form Health Survey 129 (SF-12), and Pain Catastrophizing Scale at baseline after the fifth and tenth sessions.

Results: All measures were improved throughout the first to tenth session $(P<0.05)$, though no significant differences were observed between the two groups $(P>0.05)$.

Conclusion: In summary, a significant beneficial role of acupuncture, stretching, and physiotherapy on cognitive, psychosomatic pain measures, disability index, quality of life, and catastrophizing in patients with TTH was observed.

Keywords: tension-type headache, acupuncture, physiotherapy, stretching, psychocognitive characteristics, myofascial release

\section{Introduction}

Tension-type headache (TTH) is a usual form of primary headaches with the number of disabilities referred to TTH being higher than that of migraine. ${ }^{1}$ The episodes of pain of patients with TTH have been reported to be typically bilateral, pressing, or tightening in quality, mild to moderate in intensity, and not to deteriorate with regular physical activity. ${ }^{2}$

Physical therapy and acupuncture have been used for the management of TTH, with some scientific evidence for their efficacy. There are reports where acupuncture has been observed to be clinically beneficial for patients with chronic headaches, migraines, ${ }^{3}$ and TTH. ${ }^{4}$ In addition, acupuncture has been applied worldwide for TTH prophylaxis (eg, to decrease the frequency and intensity of TTH). ${ }^{5}$ On the other hand, few data exist regarding the possible therapeutic effect of combination protocols and specifically treatment protocols integrating acupuncture with other therapeutic methods.

Moreover, physiotherapy has also been shown to treat adults with headaches, either by using hands-on techniques (eg, manual therapy, chiropractic and osteopathy 
techniques, and myofascial release techniques), ${ }^{6,7}$ stretching, or passive electrotherapy modalities. ${ }^{8}$ In pragmatic settings, however, an increased number of physiotherapists apply different therapeutic techniques to increase the analgesic effect on their patients' headaches. ${ }^{9}{ }^{910}$ This is a quite clinically relevant question worth answering in order to demonstrate if various combinations of techniques provide better improvement than others. At present, there is limited information for reports of treatment of TTH with a combination of these therapeutic techniques. Specifically, the literature lacks research data that combine physiotherapy, acupuncture, and stretching techniques. In clinical situations, the combination of such procedures is a very popular phenomenon without though established additional effectiveness.

It is the scope of this study to evaluate two clinical protocols for TTH, combining techniques such as the acupuncture, stretching, and some forms of physiotherapy, in order to initially examine if there is improvement in a number of constructs (psychocognitive, pain, etc) for each combination, and second, to compare the two combinations for differences. In the present study, "physiotherapy" includes only some myofascial release techniques and microwave diathermy. Myofascial release techniques intend to deactivate the sensi- tized myofascial trigger points (using sustained pressure) and fascia (series of strokes). ${ }^{11}$ It has been shown that fascia and myofascial trigger points therapeutic interventions decrease the local headache pain intensity and magnitude of referred pain and sensitivity. ${ }^{12,13}$ Therefore, the aim of the present study was to compare the combined effects of acupuncture, stretching, and physiotherapy on psychocognitive, somatic, quality of life, and disability characteristics in patients with TTH.

\section{Subjects and methods Study design}

A single-blind multicenter-controlled trial was adopted for the purpose of the study (Figure 1). The study protocol aimed to depict the actual clinical practice in real settings. "Sham-physiotherapy" was not included in the protocol, since the "hands-on" approach of both physiotherapy and sham-physiotherapy interventions would complicate the design. The study followed a very detailed procedure manual for all centers.

\section{Subjects}

Participants were patients with episodic TTH (n=57) with 15 or less headache days per month, who were recruited through

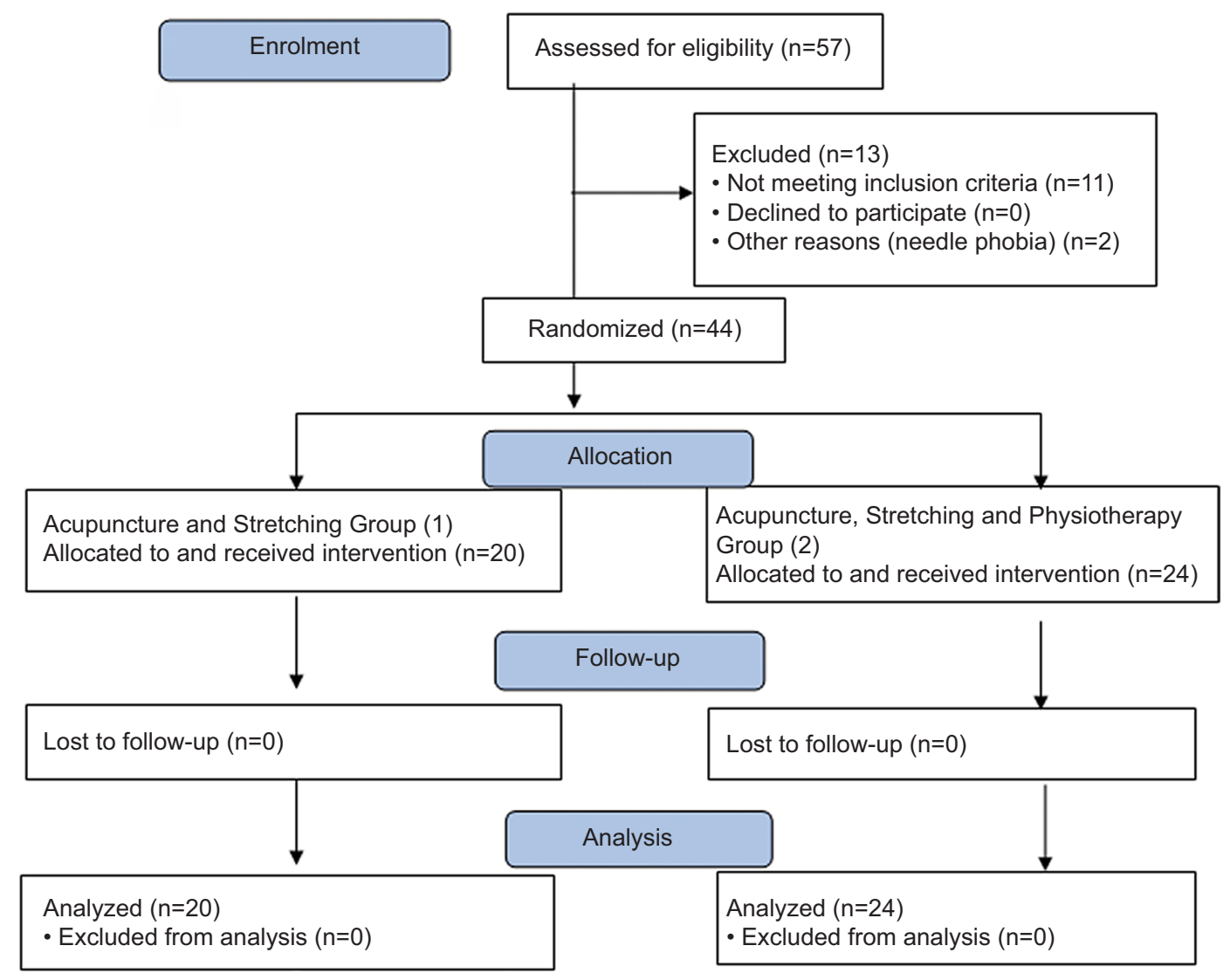

Figure I CONSORT flow diagram. 
three physiotherapy clinics. All participants volunteered in the study and were asked to provide written informed consent. A physician (either neurologist or orthopedic surgeon) set the diagnosis of the participants based on the International Classification of Headache Disorders - 3rd version (IHCD-3) criteria for TTH. Patients were considered appropriate for the study after the initial diagnosis of episodic TTH, only if they reported none of the exclusion criteria (major pathology or surgery at the neck or head; migraine headache or other officially diagnosed headache types; recent injury at the head or neck; rheumatologic or immune diseases; pregnancy; cancer pain; inability to read, understand, and write Greek; TTH prophylaxis medication within the last 12 months; recent treatment with acupuncture or physiotherapy for their headache; and needle phobia). Patients were allowed to use analgesics such as paracetamol, aspirin, and NSAIDs throughout the study, although this was recorded. Specifically, it was recorded if the participants were using no analgesics (value $=0$ ), only one type of analgesic at minimum dosage (value $=1$ ), two types of analgesics or one at max dosage (value $=2$ ), and three or more analgesics or two at maximum dosages (value $=3$ ). Thirteen participants were excluded considering the above exclusion criteria of the study resulting in a final sample of 44 participants who were randomly assigned to two experimental groups.

The basic demographic data of participants are presented in Table 1. The study was conducted according to the standards of the Declaration of Helsinki. Ethical committee approval was granted by the University of Ioannina and the Technological Educational Institute of Athens, Greece (approval: 102-7/12/2016). Part of this research project was presented as an MSc thesis of one of the authors (second author).

\section{Clinicians}

Each participant was treated by a specialized clinician who administered acupuncture and guided the self-stretching. The clinicians were blind to the identity of the group and were well experienced (ie, 2-15 years) with $>200$ hours of training in acupuncture. In order to secure consistency in the therapeutic approach, the clinicians were trained in a 1-day in-service training program emphasizing the acupuncture points, the physiotherapy modalities, the myofascial and release techniques employed (sustained pressure and deep strokes for fascia), together with the procedures of the study.

\section{Procedures}

During the first visit and before the first treatment ( $t 1)$, all patients completed a battery of questionnaires (see Instruments section), serving as baseline data. After the fifth treatment session, the patients completed once more a selected number of questionnaires $(t 2)$ to mainly record the analgesic effect of the treatment received. Straight after completing the last treatment session (tenth), for both groups, a full reassessment was performed ( $t 3$ ) including all the battery of questionnaires administered at baseline. The person administering and scoring the questionnaires was blind to the treatment group and not further implicated with the research project at any time.

Table I Descriptive statistics for the participating subjects at baseline

\begin{tabular}{|c|c|c|c|c|}
\hline \multirow[t]{2}{*}{ Variables } & \multicolumn{2}{|c|}{ Control group $(n=20)$} & \multicolumn{2}{|c|}{ Experimental group $(n=24)$} \\
\hline & Mean \pm SD & Range (min-max) & Mean \pm SD & Range (min-max) \\
\hline Age (years)* & $43.0 \pm 6.5$ & $34-54$ & $54.8 \pm 14.7$ & $29-78$ \\
\hline Height $(m)^{*}$ & $1.71 \pm 0.10$ & $1.60-1.92$ & $1.63 \pm 0.06$ & $1.54-1.80$ \\
\hline Weight (kg) & $69.5 \pm 15.0$ & $49-94$ & $64.3 \pm 11.6$ & $53-95$ \\
\hline MIDAS & $53.3 \pm 40$ & $15-136$ & $80.6 \pm 60.5$ & $5-185$ \\
\hline SFMPQ-S & $12.5 \pm 4.0$ & $6-22$ & $13.8 \pm 8.7$ & $3-30$ \\
\hline SFMPQ-A & $5.8 \pm 3.6$ & $0-12$ & $7.2 \pm 3.9$ & $0-12$ \\
\hline SFMPQ-T & $18.3 \pm 6.9$ & $6-34$ & $21.0 \pm 11.6$ & $3-40$ \\
\hline HADS-A & $11.0 \pm 3.4$ & $6-17$ & $10.7 \pm 4.0$ & $3-17$ \\
\hline HADS-D & $7.0 \pm 2.9$ & $3-14$ & $7.5 \pm 3.7$ & $2-15$ \\
\hline SF-I2 (P) & $43.0 \pm 7.7$ & $29-55$ & $39.2 \pm 9.1$ & $22-59$ \\
\hline SF-I $2(\mathrm{M})^{*}$ & $41.5 \pm 9.6$ & $24-56$ & $34.2 \pm 6.3$ & $22-46$ \\
\hline PCS Q & $27.8 \pm 15.3$ & $3-51$ & $25.9 \pm 11.5$ & $0-44$ \\
\hline Headache frequency & $8.2 \pm 3.6$ & $4-15$ & $8.1 \pm 2.8$ & $3-14$ \\
\hline
\end{tabular}

Notes: Control group: acupuncture and stretching exercise. Experimental group: acupuncture, stretching exercise, and myofascial release with diathermy. $*$ Significant differences between groups $(P<0.05)$ before treatment.

Abbreviations: HADS-A, Hospital Anxiety and Depression Scale-Anxiety; HADS-D, Hospital Anxiety and Depression Scale-Depression; MIDAS, Migraine Disability Assessment Questionnaire; PCS, Pain Catastrophizing Scale; SF-12, short Form Health Survey 12; SFMPQ-A, Short-Form McGill Pain Questionnaire-Affective; SFMPQ-S, Short-Form McGill Pain Questionnaire-Sensory. 


\section{Instruments}

The patients completed the following questionnaires at $t 1$ and $t 3$ :

1. The Greek version of the Migraine Disability Assessment Questionnaire (MIDAS), which has been validated in Greek TTH sufferers. ${ }^{14}$

2. The Greek version of the Short-Form McGill Pain Questionnaire (SFMPQ). The SFMPQ consists of 15 descriptors (11 sensory and 4 affective), which are rated on an intensity scale from 0 to 3 ( $0=$ none, $1=$ mild, $2=$ moderate, $3=$ severe). Three pain scores are derived from the sum of the intensity rank values of the words chosen for sensory (SFMPQ-S), affective (SFMPQ-A), and total descriptors (SFMPQ-T). ${ }^{15}$

3. The Visual Analog Scale (VAS) in order to describe the average intensity of pain during the last week. This 10 $\mathrm{cm}$ line, anchored with the phrases "no pain" and "worst possible pain", is a well-validated measure in chronic pain. ${ }^{16}$

4. The Greek version of the Hospital Anxiety and Depression Scale (HADS) (HAD-GR). A 14-item questionnaire (scaled 0-3) of two subscales, the 7-item HADS-anxiety (HADS-A) and the 7-item HADS-depression (HADS-D), used to assess the levels of anxiety and depression, with validity and reliability shown for the Greek version. ${ }^{17}$

5. The Greek version of short Form Health Survey 12 SF-12 (SF). The 12 items in the SF-12 are a subset of those in the SF-36; SF-12 includes 1 or 2 items from each of the eight health concepts. Thus, the SF-12 measures eight concepts commonly represented in widely used surveys: physical functioning, role limitations due to physical health problems, bodily pain, general health, vitality (energy/fatigue), social functioning, role limitations due to emotional problems, and mental health (psychological distress and psychological well-being). The scale officially has been adapted into Greek by the institute. ${ }^{18}$

6. A Greek version of the Pain Catastrophizing Scale (PCS). Pain catastrophizing is an important cognitive construct that has been linked with many aspects of the pain experience, including pain intensity, emotional distress, pain-related disability, and pain behavior. PCS has been often used to assess this construct and reflected three aspects of catastrophizing: rumination, magnification, and helplessness. The answers "never, in small degree, in mediocre degree, to a large extent, always" were marked by $0-4$ degrees, respectively. The Greek version has been shown to retain the properties of the original. ${ }^{19}$
The SFMPQ, MIDAS, HADS, SF-12, and PCS were considered as the main outcome measures for this study.

\section{Treatment options}

\section{Acupuncture}

In line with the Standards for Reporting Interventions in Clinical Trials of Acupuncture (STRICTA) 2010, ${ }^{20}$ the present trial included fundamental details about the need of acupuncture, information about needling, therapeutic modalities, clinicians' expertise, and control group. We selected the western medical approach (biomedical acupuncture) combined with some traditional Chinese medicine points, since both techniques have been shown in the literature to effectively manage TTHs. ${ }^{21}$

Twenty stainless steel needles $25 \times 0.25 \mathrm{~mm}$ in size were used in every session. The majority (15-17) of needling points were predetermined for all participants, while 3-5 points were chosen depending on symptoms. The predefined needling points were mainly locations of myofascial trigger points within the most usually affected muscles in TTH.

The needles were applied with manual stimulation for a few seconds every 5 minutes, and this procedure lasted 20 minutes. Straight after the completion of acupuncture, patients were shown the stretching protocol (see Stretching section). All needling techniques were performed within licensed practices by trained therapists, as indicated in the Clinicians section. Each needling session was vigorously recorded with all technical details.

\section{Stretching}

A stretching protocol was instructed to all patients including 3 sets of 30-second passively self-stretch of head, trapezius muscles, levator scapulae muscles, rhomboids muscles, infraspinatus, and sternocleidomastoids muscles. Left and right head rotation, protraction, and retraction head movements were additionally performed by the patients. The head rotation movements followed the same technical details as the stretching, while the protraction/retraction exercise was performed for 10 consecutive repetitions with 5 seconds holding at the end of the range of motion. A leaflet reporting the stretching exercises was provided to participants with the advice to practice the stretching session two to three times daily.

\section{Physiotherapy}

The physiotherapy referred to microwave diathermy and hands-on myofascial release techniques. The microwave diathermy was selected as a deep thermotherapy modality, 
providing a radiation field capable to produce local and deeper temperature increases. The technical parameters were a pulsed wavelength $(1: 2)$, an intensity of $75 \mathrm{~W}$, for 10 minutes.

Following the diathermy, participants were subjected to a 15-minute myofascial release protocol (ie, soft tissue and trigger point release techniques). Specifically, for the trigger points, sustained pressure was exerted for 10 seconds followed by a slight easing for further 10 seconds repeatedly, until the pain diminishes or up to the maximum time of 2 minutes, as described in a classic textbook discussing soft-tissue manipulation techniques. ${ }^{22}$ For the fascia release, a series of strokes or glides was applied to a $5-8 \mathrm{~cm}^{2}$ area within 4-5 seconds with the patient feeling some discomfort but not pain. This is known as the thumb technique. ${ }^{22}$ Based on the patient's symptoms, selected areas capable to reproduce the patient's complains were specifically treated.

\section{Statistical analysis}

A repeated measurements ANOVA (repeated measures) model was employed to detect differences in both treatment groups for all repeated measurements. Between-groups comparisons were examined with the ANOVA model. Paired $t$-test comparisons and descriptive statistics were selectively applied and calculated. For nonparametric statistics, the chi-squared test was used. The effect size was evaluated using $\eta^{2}$ as trivial $\left(\eta^{2}<0.01\right)$, small $\left(0.01 \leq \eta^{2}<0.06\right)$, moder- ate $\left(0.06 \leq \eta^{2}<0.14\right)$, and large $\left(\eta^{2} \geq 0.14\right)$ for ANOVA and Cohen's $d$ as trivial $(d<0.20)$, small $(0.20 \leq d<0.50)$, medium $(0.50 \leq d<0.80)$, and large $(d \geq 0.80)$ for $t$-test. Significance was set at $P<0.05$. SPSS v.23.0 (SPSS, Chicago, IL, USA) and GraphPad Prism v.7.0 (GraphPad Software, San Diego, CA, USA) were used in all statistical analyses.

\section{Results}

A large main effect of time on MIDAS $\left(P<0.001, \eta^{2}=0.429\right)$, SFMPQ-S $\left(P<0.001, \eta^{2}=0.760\right)$, SFMPQ-S $(P<0.001$, $\left.\eta^{2}=0.650\right)$, SFMPQ-T $\left(P<0.001, \eta^{2}=0.770\right)$, and pain intensity $\left(P<0.001, \eta^{2}=0.573\right)$ was observed, in which all scores improved across time (Figure 2). No timexgroup interaction on MIDAS ( $\left.P=0.136, \eta^{2}=0.052\right)$, SFMPQ-S $(P=0.106$, $\left.\eta^{2}=0.058\right)$, SFMPQ-A $\left(P=0.275, \eta^{2}=0.029\right)$, SFMPQ-T $\left(P=0.128, \eta^{2}=0.053\right)$, and pain intensity $\left(P=0.279, \eta^{2}=0.030\right)$ was shown indicating that the effect of time did not vary by group. The magnitude of pre- to postintervention change was larger in the experimental than in the control group for MIDAS ( $d=0.76$ vs $d=0.64$ ), pain intensity ( $d=1.79$ vs $d=1.24)$, SFMPQ-S ( $d=1.79$ vs $d=1.62)$, SFMPQ-A ( $d=1.35$ vs $d=1.15$ ), and smaller for SFMPQ-T ( $d=1.37$ vs $d=1.59$ ).

In addition, a large main effect of time on HADS-A $\left(P<0.001, \eta^{2}=0.799\right)$, HADS-D $\left(P<0.001, \eta^{2}=0.464\right)$, PCS $\left(P<0.001, \eta^{2}=0.531\right)$, SF-P $\left(P<0.001, \eta^{2}=0.485\right)$, and Short Form Health Survey 12, Mental Health Summary Subscale (SF-M) $\left(P<0.001, \eta^{2}=0.591\right)$ was observed, in which all

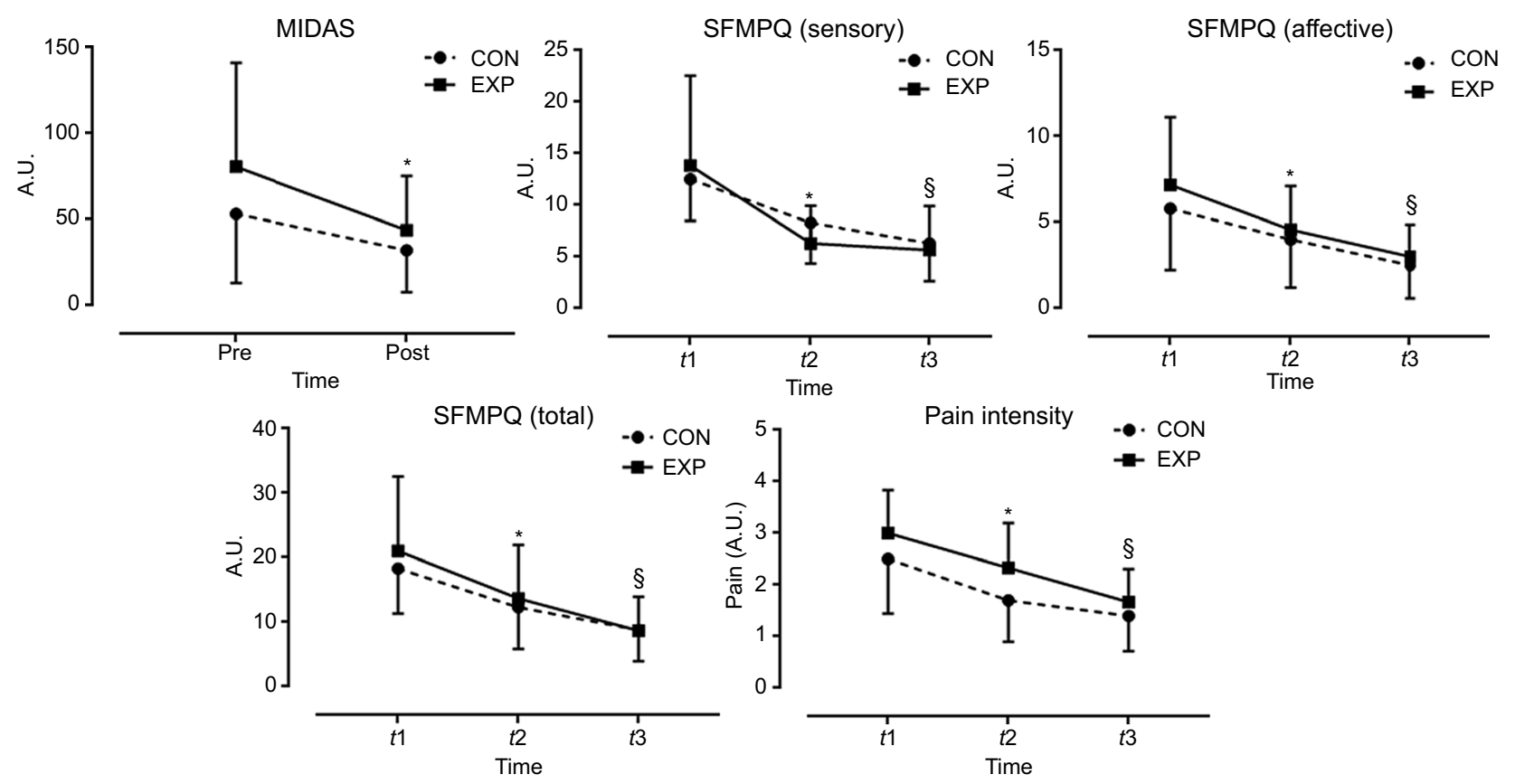

Figure 2 Effect of treatment on MIDAS, SFMPQ, and pain intensity.

Notes: *Different from $t 1$. §Different from $t \mid$ and $t 2$.

Abbreviations: A.u., anonymous units; MIDAS, Migraine Disability Assessment Questionnaire; SFMPQ, Short-Form McGill Pain Questionnaire. 


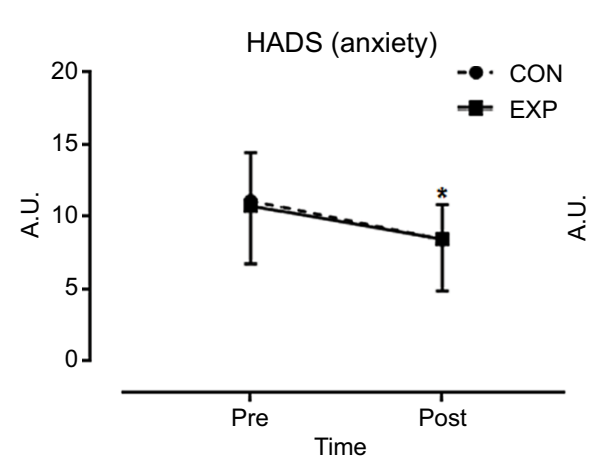

$\left.\begin{array}{r}60 \\ 50 \\ 30 \\ 20 \\ 20\end{array}\right]$

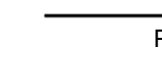

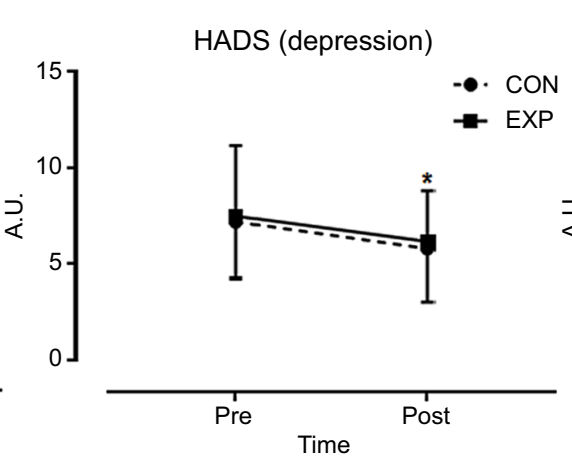

SF-P

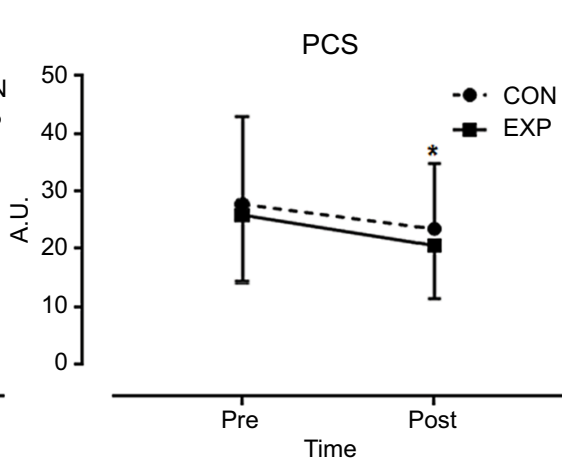

SF-M
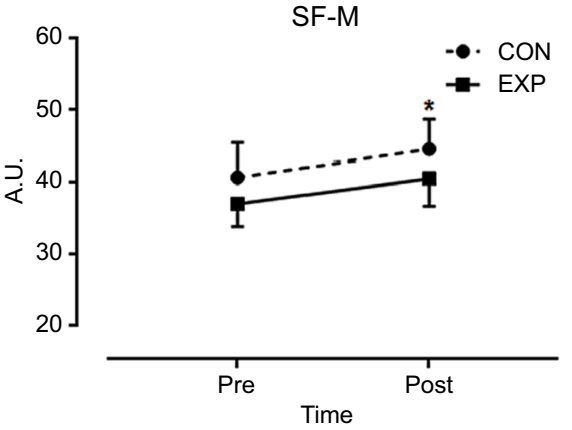

Figure 3 Effect of treatment on HADS, PCS, and SF.

Note: *Different from pretest.

Abbreviations: HADS, Hospital Anxiety and Depression Scale; PCS, Pain Catastrophizing Scale.

scores improved across time (Figure 3). No time $\times$ group interaction on HADS-A $\left(P=0.352, \eta^{2}=0.021\right)$, HADS-D $\left(P=0.884, \eta^{2}=0.001\right)$, PCS $\left(P=0.463, \eta^{2}=0.013\right)$, Short Form Health Survey 12, Physical Health Summary Subscale (SF-P) $\left(P=0.054, \eta^{2}=0.086\right)$, and SF-M $\left(P=0.739, \eta^{2}=0.003\right)$ was shown suggesting that the effect of time was similar for both treatment groups. The magnitude of pre- to postintervention change was larger in the experimental than in the control group for PCS ( $d=0.52$ vs $\mathrm{d}=0.32)$, SF-P $(d=1.14$ vs $d=0.72)$, and SF-M ( $d=1.00$ vs $d=0.85)$ and smaller for HADS-A ( $d=0.62$ vs $d=0.91)$ and HADS-D ( $d=0.41$ vs $\mathrm{d}=0.50$ ). In summary, the magnitude of change was larger in the experimental group than in the control group for 7 out of 10 outcomes under examination.

Medication use was significantly reduced for both groups from baseline to the end of treatment (acupuncture and physiotherapy group: $\chi^{2}=8.3, d f=3, P<0.05$, acupuncture group: $\left.\chi^{2}=8.0, d f=3, P<0.05\right)$.

\section{Discussion}

The present research intended to study the influence of two treatment protocols for TTH on pain sensation, functionality, quality of life, level of catastrophizing, and anxiety and depression levels. The main findings were that 1) an improvement in all measures was observed for both treatment groups (within-groups differences); 2) no timextreatment interaction effect was shown; and 3) the magnitude of the effect of treatment was larger in most of the examined variables for the experimental than for the control group.

\section{Pain measures (SFMPQ,VAS)}

The quality of pain and its intensity throughout the study for both groups continued to improve from baseline to the fifth and the tenth treatment session (SFMPQ-T, -S, -A, VAS pain intensity index). The $t 3$ measurements (after treatment) showed statistically significant improvements in pain sensation compared with $t 1$ and $t 2$ measurements for both groups. This is in accordance with the literature that shows similar effects for acupuncture and physiotherapy treatment protocols independently. ${ }^{23,24}$ No statistical significance was shown for between-groups comparisons in pain sensation. This finding may indeed be attributed to the relatively small number of participants of this study. Especially when from the related literature effect sizes and minimum clinical significance difference estimations, it is required for a primary pain outcome measure a sample size of 60 subjects per group to detect a $25 \%$ difference, at $P<0.05$ with $90 \%$ power. ${ }^{25}$ The improvement in pain measures is also dependent on the medication use. Patients in both groups significantly reduced the symptomatic analgesic use from baseline to the end of their treatment.

\section{Disability (MIDAS)}

The MIDAS score is considered an appropriate tool to assess functionality and disability in headache patients with already- 
established validity, reliability, and responsiveness in TTH Greek patients. ${ }^{14}$ It is also widely used during acupuncture treatment in headache patients to evidence improvement in their functionality. ${ }^{26}$ In this study, the improvement in disability was evidenced in both treatment groups, by the reduced MIDAS values between baseline and tenth treatment (4 weeks apart). Between-groups differences in MIDAS score were not significant indicating that neither treatment approach could show a better improvement against the other. Possibly in this case as well, the number of participating patients may be important if small effect size differences are in stake. The use of MIDAS was decided despite the fact that the two MIDAS measurements were only 1 month apart and not 3 as it is required by the authors who developed the instrument. ${ }^{27}$ The reasons for this "informality" were the appropriateness of the instrument to assess disability in TTH, especially in Greek patient samples, the convenience and easiness to complete it, and its use in the literature for periods shorter than 3 months. ${ }^{28}$ This monthly administration of MIDAS was accepted as a potential weakness of the study regarding disability assessment.

\section{Quality of life (SF-I2)}

The SF-12, a short version of the SF-36, is a valid tool to assess quality of life through its mental (SF-M) and physical health scores (SF-P). It has been reported previously that both physiotherapy and acupuncture treatments, which were used in this study, could improve quality of life measured with the SF-12. ${ }^{29,30}$ The results of this study showed no exception and both treatment protocols selected managed to reduce the SF scores, both for the mental and the physical components. No differences between treatment groups could be detected. However, the physical component of the scale had a definite trend $(P=0.054)$ for improvement in favor of the combined protocol (ie, acupuncture and physiotherapy). On the other hand, this trend could not be verified in the mental component $(P=0.739)$. The mental component score at the beginning of the study was slightly but significantly different between groups, but improved similarly for the two treatment groups, throughout the treatment showing no further clinical importance. The effect of each treatment protocol, according to this study, had a positive but similar effect on the quality of life in TTH sufferers.

\section{Anxiety, depression (HADS), and catastrophising (PCS)}

A significant improvement in anxiety, depression, and catastrophizing levels was noted for all patients in the study, independently of the treatment followed (HADS_AnxietyScore
- HADS_DepressionScore - PCS_score). No differences between treatment groups could be noted. Apart from the small number of subjects, the literature has also shown that due to the complex central phenomena in chronic pain states, both physiological and psychological effects of hands-on treatments may not be rapid, and it may take 6 weeks of time or more to establish significant differences. ${ }^{31}$ It remains the clinical question if another study with extended follow-up periods could establish a between-groups statistically significant difference.

All baseline anxiety, depression, and catastrophizing scores within groups were severely reduced at the tenth treatment confirming what was known in the literature. When the pain is reduced, the psychological status of the patient improves as well. Numerous paradigms in the literature support these findings. ${ }^{24,31-33}$ Especially, when using hands-on techniques (eg, myofascial release and needling), a number of substances, such as the neuropeptides serotonin and norepinephrine, which are substantial mediators both in pain and psychological manifestations (anxiety, depression, catastrophizing, etc), are modified centrally. This central effect of hands-on techniques further stimulates the parasympathetic branch of the autonomic nervous system, facilitating psychological distress reduction. ${ }^{34}$ The psychological manifestations are such a common ailment in chronic pain states and in TTH that a positive modification through acupuncture and myofascial release would benefit both pain and psychological status of the patients. ${ }^{32}$

An interesting comment for this study is the design selected where acupuncture and stretching were used in both groups and physiotherapy in one. Since acupuncture ${ }^{3-5}$ and physiotherapy ${ }^{6-9}$ are considered beneficial means on their own for headache patients in the literature, it might be interesting to wonder what would be the results if the design was vice versa, with physiotherapy in both groups and acupuncture only in one? This question would need an additional group in the study to answer it. However, if the results were equally beneficial, then it could be assumed that the two measures may have similar analgesic efficacy and when one is applied, the other cannot further improve the results. This assumption reflects the clinical opinion of the authors on the efficacy of the above therapies, although it remains to be explicitly shown in a future study.

\section{Conclusions}

In summary, both treatment groups (acupuncture and stretching vs acupuncture, stretching, and physiotherapy) for episodic TTH headaches showed statistically significant 
improvement from a protocol of acupuncture, stretching, and physiotherapy. Cognitive and psychosomatic pain measures, disability index, quality of life, and catastrophizing were improved for both groups during either treatment protocol, failing though to show statistically significant differences between groups. In clinical terms, the combination of physiotherapy with acupuncture and stretching or acupuncture and stretching alone are recommended both for the improvement of the physical and affective components of pain but also of the psychological status of the sufferer.

\section{Acknowledgments}

We would like to acknowledge the assistance of Mr Aggourakis Manolis and the personnel in the PHYSIOPAIN group practices in data collection.

\section{Disclosure}

The authors report no conflicts of interest in this work.

\section{References}

1. Stovner L, Hagen K, Jensen R, et al. The global burden of headache: a documentation of headache prevalence and disability worldwide. Cephalalgia. 2007;27(3):193-210.

2. Headache Classification Subcommittee of the International Headache Society. The International Classification of Headache Disorders: 2nd edition. Cephalalgia. 2004;24(Suppl 1):9-160.

3. Vickers AJ, Rees RW, Zollman CE, et al. Acupuncture of chronic headache disorders in primary care: randomised controlled trial and economic analysis. Health Technol Assess. 2004;8(48):1-35.

4. Linde K, Allais G, Brinkhaus B, et al. Acupuncture for the prevention of tension-type headache. Cochrane Database Syst Rev. 2016;4:CD007587.

5. Burke A, Upchurch DM, Dye C, Chyu L. Acupuncture use in the United States: findings from the National Health Interview Survey. J Altern Complement Med. 2006;12(7):639-648.

6. Bryans R, Descarreaux M, Duranleau M, et al. Evidence-based guidelines for the chiropractic treatment of adults with headache. J Manipulative Physiol Ther. 2011;34(5):274-289.

7. Haas M, Bronfort G, Evans R, et al. Dose-response and efficacy of spinal manipulation for care of cervicogenic headache: a dual-center randomized controlled trial. Spine J. Epub 2018 Feb 23.

8. Tella BA, Unubum EV, Danesi MA. The effect of TENS on selected symptoms in the management of patients with chronic tension type headache: a preliminary study. Nig $Q J$ Hosp Med. 2008;18(1): 25-29.

9. France S, Bown J, Nowosilskyj M, Mott M, Rand S, Walters J. Evidence for the use of dry needling and physiotherapy in the management of cervicogenic or tension-type headache: a systematic review. Cephalalgia. 2014;34(12):994-1003.

10. Ohlsen BA. Combination of acupuncture and spinal manipulative therapy: management of a 32-year-old patient with chronic tension-type headache and migraine. J Chiropr Med. 2012;11(3):192-201.

11. Alonso-Blanco C, de-La-Llave-Rincón AI, Fernández-de-Las-Peñas C. Muscle trigger point therapy in tension-type headache. Expert Rev Neurother. 2012;12(3):315-322.

12. Chatchawan U, Eungpinichpong W, Sooktho S, Tiamkao S, Yamauchi J. Effects of Thai traditional massage on pressure pain threshold and headache intensity in patients with chronic tension-type and migraine headaches. J Altern Complement Med. 2014;20(6):486-492.
13. Toro-Velasco C, Arroyo-Morales M, Fernández-de-Las-Peñas C, Cleland JA, Barrero-Hernández FJ. Short-term effects of manual therapy on heart rate variability, mood state, and pressure pain sensitivity in patients with chronic tension-type headache: a pilot study. JManipulative Physiol Ther. 2009;32(7):527-535.

14. Georgoudis G, Parasxou A, Panagopoulou X, Papathanasiou G. 816 MIDAS questionnaire in a Greek sample of headache patients: validity, reliability, responsiveness and psychometrics. Eur J Pain. 2009;13:S234.

15. Georgoudis G, Oldham JA. Anxiety and depression as confounding factors in cross-cultural pain research studies: validity and reliability of a Greek version of the Hospital Anxiety \& Depression Scale. Physiotherapy. 2001;87(2):92-93.

16. Ferreira-Valente MA, Pais-Ribeiro JL, Jensen MP. Validity of four pain intensity rating scales. Pain. 2011;152(10):2399-2404.

17. Michopoulos I, Douzenis A, Kalkavoura C, et al. Hospital Anxiety and Depression Scale (HADS): validation in a Greek general hospital sample. Ann Gen Psychiatry. 2008;7:4.

18. Kontodimopoulos N, Pappa E, Niakas D, Tountas Y. Validity of SF-12 summary scores in a Greek general population. Health Qual Life Outcomes. 2007;5:55.

19. Papaioannou M, Skapinakis P, Damigos D, Mavreas V, Broumas G, Palgimesi A. The role of catastrophizing in the prediction of postoperative pain. Pain Med (Malden, Mass). 2009;10(8):1452-1459.

20. Macpherson H, Altman DG, Hammerschlag R, et al. Revised STandards for Reporting Interventions in Clinical Trials of Acupuncture (STRICTA): extending the CONSORT statement. J Evid Based Med. 2010;3(3):140-155.

21. Larner AJ. Guidelines for the management of headache in primary care: are they being used? J Headache Pain. 2005;6(5):420-421.

22. Chaitow L. Soft-Tissue Manipulation. 3rd ed. Vermont: Rochester; 1988.

23. Endres HG, Böwing G, Diener HC, et al. Acupuncture for tension-type headache: a multicentre, sham-controlled, patient-and observer-blinded, randomised trial. J Headache Pain. 2007;8(5):306-314.

24. Moraska AF, Stenerson L, Butryn N, Krutsch JP, Schmiege SJ, Mann JD. Myofascial trigger point-focused head and neck massage for recurrent tension-type headache: a randomized, placebo-controlled clinical trial. Clin J Pain. 2015;31(2):159-168.

25. Julious SA. Sample sizes for clinical trials with normal data. Stat Med. 2004;23(12):1921-1986.

26. Facco E, Liguori A, Petti F, et al. Traditional acupuncture in migraine: a controlled, randomized study. Headache. 2008;48(3):398-407.

27. Stewart WF, Lipton RB, Kolodner KB, Sawyer J, Lee C, Liberman JN. Validity of the Migraine Disability Assessment (MIDAS) score in comparison to a diary-based measure in a population sample of migraine sufferers. Pain. 2000;88(1):41-52.

28. Plank S, Goodard JL, Pasierb L, Simunich TJ, Croner JR. Standardized set-point acupuncture for migraines. Altern Ther Health Med. Nov. 2013;19(6):32-37.

29. Couto C, de Souza IC, Torres IL, Fregni F, Caumo W. Paraspinal stimulation combined with trigger point needling and needle rotation for the treatment of myofascial pain: a randomized sham-controlled clinical trial. Clin J Pain. 2014;30(3):214-223.

30. Espí-López GV, Rodríguez-Blanco C, Oliva-Pascual-Vaca A, MolinaMartínez F, Falla D. Do manual therapy techniques have a positive effect on quality of life in people with tension-type headache? A randomized controlled trial. Eur J Phys Rehabil Med. 2016;52(4):447-456.

31. Moraska A, Chandler C. Changes in psychological parameters in patients with tension-type headache following massage therapy: a pilot study. J Man Manip Ther. 2009;17(2):86-94.

32. Balottin U, Fusar Poli P, Termine C, Molteni S, Galli F. Psychopathological symptoms in child and adolescent migraine and tension-type headache: a meta-analysis. Cephalalgia. 2013;33(2):112-122.

33. Cabýoglu MT, Ergene N, Tan U. The mechanism of acupuncture and clinical applications. Int J Neurosci. 2006;116(2):115-125.

34. Cowen VS, Burkett L, Bredimus J, et al. A comparative study of Thai massage and Swedish massage relative to physiological and psychological measures. J Bodyw Mov Ther. 2006;10(4):266-275. 
The Journal of Pain Research is an international, peer reviewed, open access, online journal that welcomes laboratory and clinical findings in the fields of pain research and the prevention and management of pain. Original research, reviews, symposium reports, hypothesis formation and commentaries are all considered for publication.
The manuscript management system is completely online and includes a very quick and fair peer-review system, which is all easy to use. Visit http://www.dovepress.com/testimonials.php to read real quotes from published authors. 